\title{
Weitere Studien über das physiologische Verhalten von I-, d- und dl-Suprarenin.
}

II. Mitteilung.

Von

Emil Abderhalden und Friedrich Thies.

Mit einer Tafel.

(Aus dem physiologischen Institut der tierärztlichen Hochschule, Berlin.)

(Der Redaktion zugegangen am 21. Januar 1909.)

Der eine von uns ${ }^{1}$ ) hat kürzlich in Gemeinschaft mit Franz Müller mitgeteilt, daß die Komponenten des dl-Suprarenins ein verschiedenes Verhalten gegenüber dem Blutdrucke zeigen. Während l-Suprarenin von bestimmten Dosen an eine ausgesprochene Erhöhung des Blutdruckes bewirkt, läßt sich beim d-Suprarenin bei Anwendung gleicher und auch größerer Mengen kaum ein Einfluß feststellen. Die Wirkung des racemischen Suprarenins ist, ganz im Einklang mit dieser Beobachtung, abhängig von der Menge der l-Komponente, d. h. die doppelte Menge dl-Suprarenin wirkt so stark, wie die einfache Menge l-Suprarenin. Das d-Suprarenin war in den angewandten Dosen nicht vollständig wirkungslos. Sein Einfluß war jedoch so gering, daß wir wohl annehmen dürfen, da $\beta$ dem $d$-Suprarenin noch Spuren von l-Suprarenin angehaftet haben. Das optische Verhalten beider Komponenten läßt zwar auf eine gute Trennung beider Komponenten schließen. Das verwendete l-Suprarenin zeigte in der berechneten Menge n-Salzsäure gelöst $[\alpha]_{20}^{\mathrm{D}}$ $=-50,40^{\circ}$ und die d-Komponente $[\alpha]_{20^{\circ}}^{\mathrm{D}}=+50,49^{\circ}$. Das physiologische Experiment gibt hier offenbar schärfere Resultate als die optische Untersuchung.

1) Emil Abderhalden u. Franz Müller, Über das Verhalten des Blutdruckes nach intravenöser Einführung von l-, d- und dl-Suprarenin. Diese Zeitschrift, Bd. LVIII, S. 185, 1908. 


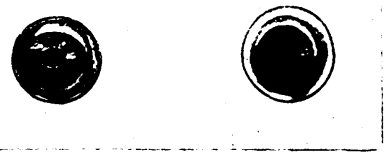

Vor
Zusatz von l-Suprarenin $(1: 1000)$.

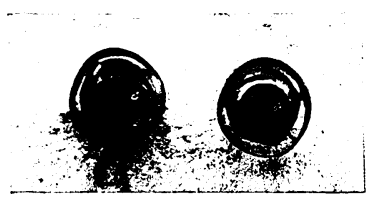

Vor

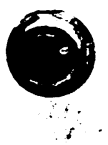

Nach

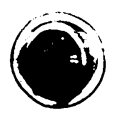

(Kontrollauge)

Zusal\% von d-Suprarenin $(1: 5())()$

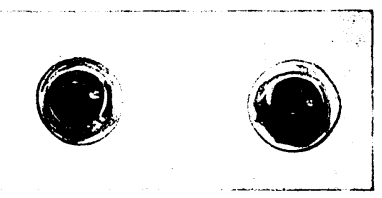

Vor

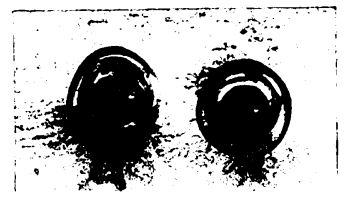

Nach

Kontrollauge)

Zusat\% von dl-Suprarenin (1: jo0)

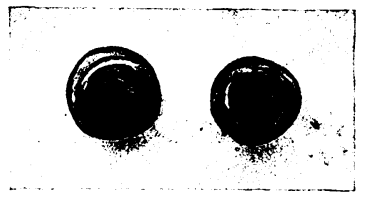

Vor

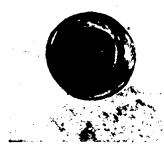

Nach

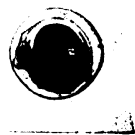

(Kontrollange)

Zusatz von dl-Suprarenin (1: $1000 \%$.

Hoppe-Seyler's Zeitschrift für physiologische Chemie. Band IX, Tafe? 1. Zu *Abderhalden und Thies, Weitere Studien über das phsiologische Verhalten von l-, d- und dl-Suprarenin". Il. 
Brought to you by | Purdue University Libl Authenticated

Download Date | 5/31/15 3:50 PM 
Wir haben nun diese Versuche auf das Verhalten der Pupille beim Frosche gegenüber l-, d- und dl-Suprarenin ausgedehnt und ferner den Einfluß des racemischen Suprarenins sowie seiner Komponenten auf den Zuckerstoffwechsel beim Kaninchen geprüft. Die Resultate waren die folgenden.

1. d-Suprarenin bewirkt in Mengen, bei denen l-Suprarenin eine ausgesprochene Pupillenerweiterung am Froschauge herbeiführt, keine oder doch nur eine geringfügige Erweiterung der Pupille. dl-Suprarenin wirkt seinem Gehalte an l-Suprarenin entsprechend.

2. d-Suprarenin ruft in Dosen, die bei Anwenung von l-Suprarenin Glukosurie bewirken, keine Zuckerausscheidung hervor. dl-Suprarenin wirkt auch hier seinem Gehalte an l-Suprarenin entsprechend.

Es kommt somit die Konfiguration des Suprarenins bei all diesen Versuchen in ganz ausgesprochener Weise zum Ausdruck. Wir haben ganz ähnliche Beziehungen, wie sie bereits Cushny ${ }^{1}$ ) für das Atropin resp. das l- und d-Hyoscyamin nachgewiesen hat. Da nun beim Suprarenin, wie die unten mitgeteilten Versuche sehr deutlich beweisen, die Konfiguration einen so ausgesprochenen Einfluß auf die physiologische Wirkung dieser Verbindung besitzt, so unterliegt es keinem Zweifel, daß auch diejenige Substanz, auf die das Suprarenin wirkt, Gruppen besitzen muß, die einen ganz spezifischen Aufbau besitzen. Wir haben hier in gewissem Sinne ganz ähnliche Verhältnisse vor uns, wie bei den spezifisch wirkenden Fermenten. Auch diese wirken nur auf Verbindungen von ganz bestimmter Konfiguration. Auch für die Toxine dürfen wir ganz ähnliche Beziehungen vermuten. Während wir jedoch bei den Fermenten in vielen Fällen, vor allem dank den Untersuchungen Emil Fischers, genau über die Konfiguration des Substrates, auf das ein bestimmtes Ferment eingestellt ist, unterrichtet sind, fehlt uns vorläufig jeder Einblick in den Auf-

1) Arthur R. Cushny, Atropine and the hyoscyanines - a study of the action of optical isomeres. J. of Physiologie, Bd. XXX, S. 176, 1904. 
bau der Fermente. Wir schließen indirekt, daß das Ferment einen Bau besitzen müsse, der enge Beziehungen zu dem Substrat, auf das es eingestellt ist, aufweist. Bei unseren Versuchen kennen wir nun zwar die Konfiguration der Komponenten des l- und d-Suprarenins noch nicht, wohl aber sind wir durch die von Stolz $^{1}$ ) und E. Friedmann ${ }^{2}$ ) ausgeführte Synthese genau über die Struktur des dl-Suprarenins aufgeklärt. Hier ist der gewissermaßen angreifende Körper bekannt, dagegen das Substrat nach seinem Aufbau vollständig unbekannt. Nach all unseren Vorstellungen über die Wirkungsweise der Fermente und der Toxine dürfen wir wohl auch hier, wie schon erwähnt, vermuten, daß das Substrat, auf welches das l-Suprarenin eingestellt ist, Beziehungen irgend welcher Art in seinem Aufbau zum wirksamen Prinzip - dem l-Suprarenin - besitzen muß. Derartigen Verbindungen nachzuforschen, ist eine reizvolle Aufgabe weiterer Untersuchungen. Nach all unseren Kenntnissen muß dieses Substrat entweder im Nervensystem und zwar in den Sympathicusfasern vorhanden sein oder aber in den von ihnen innervierten Zellen. Derartige Beziehungen von Verbindungen mit ganz bestimmter Konfiguration und ganz bestimmter Wirkung zu Bausteinen von Geweben erscheinen uns geeignet, neue Bahnen zur Erforschung der Wechselbeziehungen der einzelnen Organe unter einander zu eröffnen.

Der Befund, daß das verschiedene Verhalten von l- und d-Suprarenin in gleicher Weise bei der Beeinflussung des Blutdruckes, der Pupillenweite und des Zuckerstoffwechsels zum Ausdruck kommt, macht es im höchsten Grade wahrscheinlich, daß der Angriffspunkt des l-Suprarenins in allen Fällen der gleiche ist.

Wir beabsichtigen, noch weitere Versuche mit l-, $d-$ und dl-Suprarenin und anderen optisch-aktiven Substanzen mit ausgesprochener Wirkung anzustellen.

1) Friedrich Stolz, Über Adrenalin und Alkylaminoacetobrenzkatechin. Ber. d. Deutsch. chem. Ges., Bd. XXXVII, S. 41, 1904.

$\left.{ }^{2}\right)$ E. Friedmann, Zur Kenntnis des Adrenalins (Suprarenins). Hofmeisters Beiträge, Bd. VI, S. 92, 1904, und Die Konstitution des Adrenalins. Hofmeisters Beiträge, Bd. VIII, S. 94, 1906. 
Über das physiologische Verhalten von l-, d- und dl-Suprarenin. II. 25

\section{Experimenteller Teil.}

1. Versuche an Kaninchen.

\begin{tabular}{|c|c|c|c|c|c|c|}
\hline Datum & $\begin{array}{c}\text { Kör- } \\
\text { per- } \\
\text { ge- } \\
\text { wicht } \\
\text { in g }\end{array}$ & $\begin{array}{l}\text { Futter- } \\
\text { menge }\end{array}$ & $\begin{array}{l}\text { Harn- } \\
\text { menge } \\
\text { in } \mathrm{ccm}\end{array}$ & $\mid \begin{array}{c}\mathrm{N}- \\
\text { Gehalt } \\
\text { des } \\
\text { Harnes } \\
\text { in } \mathrm{g}\end{array}$ & $\left|\begin{array}{c}\text { Zucker- } \\
\text { gehalt } \\
\text { des } \\
\text { Harnes } \\
\text { in } g\end{array}\right|$ & Bemerkungen \\
\hline 8. I./09 & 2000 & $\begin{array}{r}30 \mathrm{~g} \text { Hafer } \\
120 \text {. Kohl }\end{array}$ & 265 & 0,81 & 0 & - \\
\hline 9. I./09 & 1950 & $\begin{aligned} 30 & \text {. Hafer } \\
200 & \gg \text { Kohl }\end{aligned}$ & 130 & 0,61 & 0 & $\begin{array}{l}0,0001 \mathrm{~g} \text { l-Supra- } \\
\text { renin subkutan }\end{array}$ \\
\hline 10. I./09 & 1920 & $\begin{aligned} 30 & \gg \text { Hafer } \\
250 & \gg \text { Kohl }\end{aligned}$ & 170 & 1,5 & 0 & $\begin{array}{l}0,0002 \text { g l-Supra- } \\
\text { renin subkutan }\end{array}$ \\
\hline 11. I./09 & 1955 & $\begin{aligned} 60 & \gg \text { Hafer } \\
275 & \gg \text { Kohl }\end{aligned}$ & 220 & 1,12 & 1,75 & $\begin{array}{l}0,0004 \text { g l-Supra- } \\
\text { renin subkutan }\end{array}$ \\
\hline 12. I./09 & 1990 & $\begin{aligned} 50 & \text {. Hafer } \\
250 & \text {. Kohl }\end{aligned}$ & 154 & 0,93 & 0 & - \\
\hline 13. I./09 & 1950 & $\begin{aligned} 50 & , \text { Hafer } \\
250 & , \text { Kohl }\end{aligned}$ & 225 & 1,15 & 0 & $\begin{array}{l}0,0004 \mathrm{~g} \text { d-Supra- } \\
\text { renin subkutan }\end{array}$ \\
\hline 14. I./09 & 2000 & $\begin{aligned} 50 & \text {. Hafer } \\
250 & \text {. Kohl }\end{aligned}$ & 105 & 0,95 & 0,54 & $\begin{array}{l}0,0004 \text { g l-Supra- } \\
\text { renin subkutan }\end{array}$ \\
\hline 15. I /09 & 2000 & $\begin{array}{r}50 \text {. Hafer } \\
250 \text {. Kohl }\end{array}$ & 145 & 1,23 & 0 & - \\
\hline 16. I./09 & 2030 & $\begin{aligned} 50 & =\text { Hafer } \\
250 & =\text { Kohl }\end{aligned}$ & 85 & 0,97 & 0,2 & $\underset{\text { renin }}{0,0008 \mathrm{~g} \mathrm{dl-Supra-}}$ \\
\hline 17. I./09 & 2000 & $\begin{aligned} 50 & \text {. Hafer } \\
250 & \text {. Kohl }\end{aligned}$ & 186 & 1,34 & 0 & - \\
\hline 18. I./09 & 2030 & $\begin{aligned} 50 & \text {. Hafer } \\
250 & \text {. Kohl }\end{aligned}$ & 140 & 0,96 & 0 & $\underset{\text { renin }}{0,0008 \mathrm{~g} \mathrm{~d} \text { d-Supra- }}$ \\
\hline 19. I./09 & 2000 & $\begin{aligned} 50 & \text {. Hafer } \\
250 & \text {. Kohl }\end{aligned}$ & 130 & 0,87 & 0 & - \\
\hline 20. 1./09 & 1960 & $\begin{aligned} 50 & \rightarrow \text { Hafer } \\
250 & : \text { Kohl }\end{aligned}$ & 200 & 1,19 & 0,59 & $\begin{array}{c}0,0004 \text { g l-Supra- } \\
\text { renin }\end{array}$ \\
\hline
\end{tabular}

$\mathrm{Zu}$ diesen Versuchen ist $\mathrm{zu}$ bemerken, daß der Zuckergehalt des Urins zunächst qualitativ mit Hilfe der Trommerschen Probe festgestellt wurde. In jedem Falle wurde der Harn noch auferdem polarisiert und nach Bertrand und Fehling die quantitative Zuckerbestimmung durchgeführt und zwar auch dann, wenn der qualitative Zuckernachweis negativ verlaufen 
war. Die mit Hilfe der Polarisation gefundenen Werte waren alle etwas höher, als die in der obigen Tabelle angeführten. Alle mitgeteilten Zuckerwerte entsprechen den mit Hilfe der Bertrandschen Methode gefundenen. Das erhaltene Resultat ist ganz eindeutig. Erwähnen wollen wir noch, daß auch wir beobachteten, daß die intravenöse Zufuhr von l-Suprarenin selbst in Dosen, die bei subkutaner Eingabe zu Glukosurie führen, keine Zuckerausscheidung bewirkt.

\section{Versuche an Froschaugen.}

Die Versuche wurden in der Weise ausgeführt, daß ausgeschnittene, auf kleine Glastrichter gebrachte Froschaugen belichtet wurden. Die Pupillenweite wurde dann gemessen und nunmehr ein Tropfen Suprareninlösung ${ }^{1}$ ) aufgeträufelt. In jedem einzelnen Versuche verwendeten wir ein Augenpaar. Das eine Auge (B) diente zur Kontrolle. An Stelle der Suprareninlösung wurde diesem ein Tropfen physiologischer Kochsalzlösung zugefügt.

\section{Versuch 1.}

Auge A. Aufträufeln eines Tropfens einer Lösung von 1-Suprarenin 1: 10000 .

a) Größte Länge der Pupille bei Beginn des Versuches $4 \mathrm{~mm}$.

b) B Breite ,

a) Länge

b) $>$ Breite

a) $\triangleright$ Länge $>, \quad, 40$,

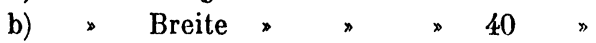

a) > Länge » $>>50$

b) $>$ Breite

Auge B. Kontrollauge.

a) Größte Länge der Pupille bei Beginn des Versuches $3 \frac{3}{4} \mathrm{~mm}$.

b) $>$ Breite $\gg, ~ \gg, 2^{3 / 4}$,

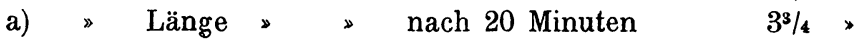

b) $>$ Breite $>>20>23 / 4$,

a) > Länge > $>40$ > $43 / 4$,

b) $>$ Breite $>40,21 / 2$,

a) > Länge » > $>50$, $33 / 4$,

b) $>$ Breite > $>50$ 2 $21 / 4$

1) Das Suprarenin wurde in allen Versuchen in der berechneten Menge Salzsäure gelöst. 
Über das physiologische Verhalten von l-, d- und dl-Suprarenin. II. 27

Versuch 2 .

Auge A. 1 Tropfen 1-Suprarenin $(1: 10000)$ aufgeträufelt.

a) Größte Länge der Pupille bei Beginn des Versuches $4^{1 / 2} \mathrm{~mm}$.

b) , Breite, , , , , $2^{2 / 3}$,

a) Länge, , nach 30 Minuten $5^{1 / 2}$,

b) D Breite ,, 30 " $4^{3 / 4}$,

a) > Länge, $>\quad 60>51 / 2$,

b) > Breite > $>60$, 5,0 ,

Das Kontrollauge zeigte folgende Werte:

a) Bei Beginn des Versuches a) $4^{1 / 2} \mathrm{~mm}$ und b) $2^{2 / 3} \mathrm{~mm}$.

Nach 30 Minuten betrugen die entsprechenden Werte $41 / 8 \mathrm{~mm}$ und $2^{2 / 3} \mathrm{~mm}$ und nach 60 Minuten $4 \mathrm{~mm}$ und $2^{2} / \mathrm{mm}$.

Versuch 3 .

Auge A. dl-Suprarenin (1:5000).

a) bei Beginn des Versuches $4 \mathrm{~mm}$

b) , , 3 ,

a) nach 30 Minuten $\quad 4^{1 / 2}$,

b) $30,44^{1 / 4}$,

a) 60 , $4^{1 / 2}$,

b) $\$ 60,4 \frac{1 / 2}{2}$

Auge B (Kontrolle).

$4 \mathrm{~mm}$

$2^{1 / 2}$,

$31 / 2$,

$2^{1 / 2}$,

$3^{1 / 2}$.

$2^{1 / 2}$,

Versuch 4 .

Auge A. dl-Suprarenin $(1: 10000)$.

a) bei Beginn des Versuches $3 \mathrm{~mm}$

b) , , 2 ,

a) nach 30 Minuten $\quad 4^{1 / 2}$,

b) 30 , 3 ,

a) 60 . $4^{1 / 3}$,

b) 60 \$ $32 / 3$,

Auge B (Kontrolle).

Versuch 5 .

Auge A. d-Suprarenin $(1: 10000)$.

a) bei Beginn des Versuches $3 \mathrm{~mm}$

b) $>\quad>1 \frac{1}{3}$,

a) nach 30 Minnten 3 ,

b) 30 , $1^{3 / 4}$,

a) 60 , 3 ,

b) $60,1^{3 / 4}$,

Auge B (Kontrolle).

$3 \mathrm{~mm}$

$1^{3 / 4}$,

3 *

$1^{1 / 2}$,

3 ,

$1^{1 / 2}$, 
28 Abderhalden und Thies, Über l-, d- und dl-Suprarenin.

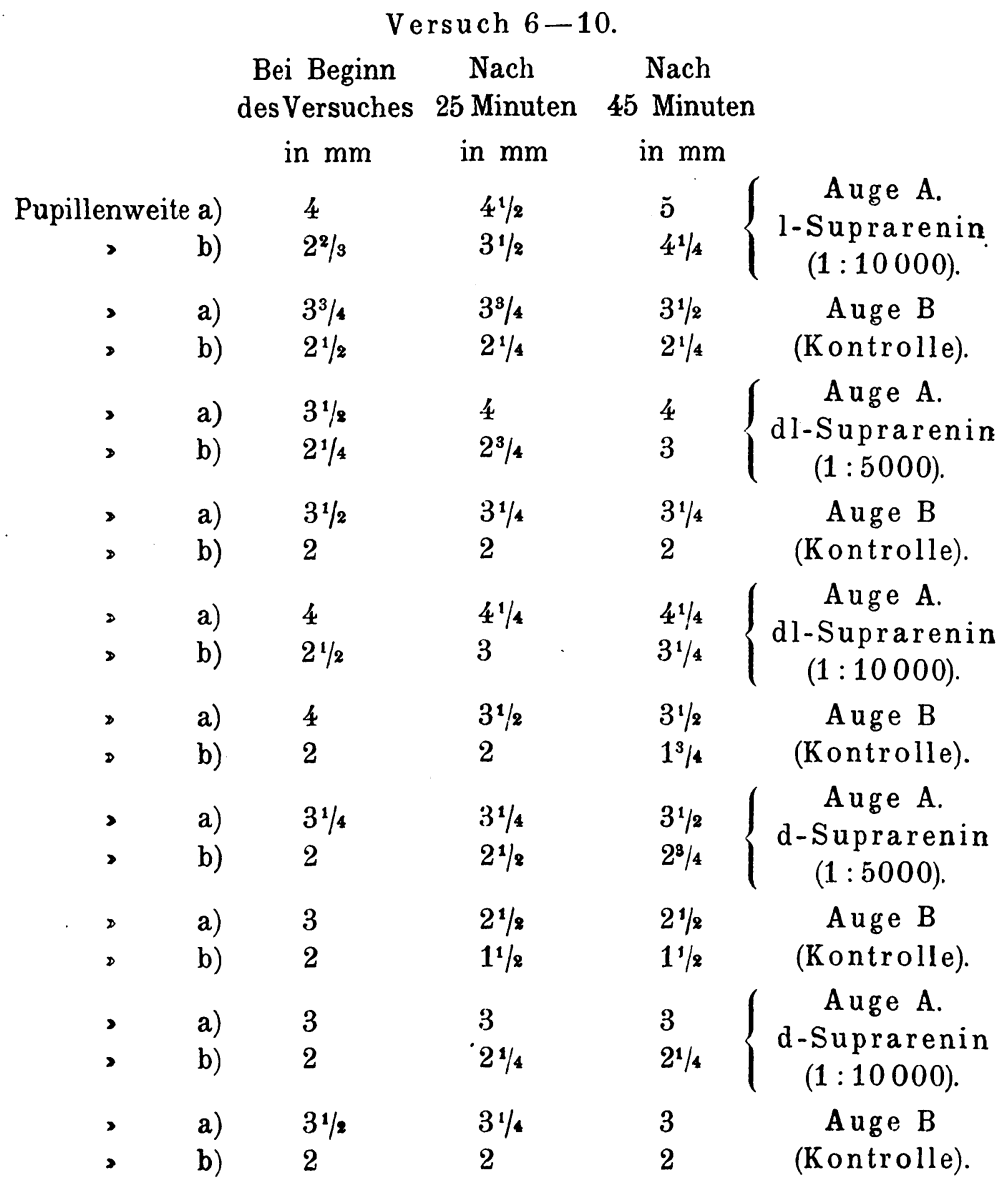

Die folgenden Abbildungen zeigen den Unterschied in der Wirkungsweise von l-, d- und dl-Suprarenin sehr deutlich. 